Volume 2

Issue 4 -- Integrative Medicine

Article 21

$11-20-2015$

\title{
Stent Thrombosis: Regional Prevalence, Risk Factors, and Outcomes
}

Andrew M. Ayers

Chi C. Cho

Robyn Shearer

M. Fuad Jan

Anjan Gupta

Follow this and additional works at: https://aah.org/jpcrr

Part of the Cardiology Commons, Cardiovascular Diseases Commons, and the Cardiovascular System Commons

\section{Recommended Citation}

Ayers AM, Cho CC, Shearer R, Jan M, Gupta A. Stent Thrombosis: Regional Prevalence, Risk Factors, and Outcomes. J Patient Cent Res Rev 2015;2:208-209. http://dx.doi.org/10.17294/2330-0698.1231

Published quarterly by Midwest-based health system Advocate Aurora Health and indexed in PubMed Central, the Journal of Patient-Centered Research and Reviews (JPCRR) is an open access, peer-reviewed medical journal focused on disseminating scholarly works devoted to improving patient-centered care practices, health outcomes, and the patient experience. 
oncogene that is overexpressed in $25-30 \%$ of breast cancers. In combination with first-line therapy, trastuzumab resulted in significant improvement in survival outcomes for those with HER2-positive metastatic breast cancer. Due to its improvement in outcome and prolonged survival, trastuzumab has been established as standard of care in both adjuvant and metastatic settings. However, along with common adverse events, trastuzumab has been found to be associated with cardiotoxicity. An estimated $1-4 \%$ of patients treated with trastuzumab will develop heart failure and $\sim 10 \%$ of patients will experience a reduction in left ventricular ejection fraction (LVEF). Many studies have published on the risk factors of trastuzumab-induced cardiotoxicity (TIC), with some discrepancy. Whereas one study found that of all risk factors accounted for (age, hypertension, LVEF, radiotherapy) only age was significantly associated with TIC, another found that LVEF was the sole factor, and others found that a combination of these were indicative of TIC.

Purpose: This paper aims to consolidate the data and identify potential risk factors from combined data.

Methods: A computer-based literature search using MEDLINE database was executed using the keywords trastuzumab/Herceptin, risk factors, outcomes, cardiac, cardiotoxicity, cardiomyopathy, LVEF and chemotherapy. Only prospective/retrospective human studies were included, with additional studies excluded if they reported a baseline LVEF $>68$, a cohort $<50$ patients, and/or results were not stratified based on cadiotoxic events.

Results: Data was collected from 17 articles, capturing 6,527 patients. A familial history of cardiac disease (odds ratio [OR]: $3.31,95 \%$ confidence interval [CI]: 1.80-6.08; $\mathrm{P}<0.01$ ), diagnoses of hypertension (OR: $1.61,95 \% \mathrm{CI}$ : 1.14-2.26; $\mathrm{P}<0.01$ ), diabetes (OR: 1.62, 95\% CI: 1.1-2.38; $\mathrm{P}=0.014$ ), and previous anthracycline use (OR: 2.14, 95\% CI: $1.17-3.92 ; \mathrm{P}=0.013)$ were all shown to be associated with TIC. Age $(\mathrm{P}=0.013)$ also was a risk factor.

Conclusion: Additional measures need to be set in place for monitoring cardiac performance in women treated with trastuzumab. Being aware of the potential risk factors along with careful attention to symptoms/LVEF can hopefully minimize the occurrence of TIC in this population.

\section{Delirium Recognition in Hospitalized Older Patients: A Quality Improvement Project}

\section{Jodi Punke, Ariba Khan, Michael L. Malone}

\section{Department of Geriatrics, Aurora Sinai Medical Center and Aurora UW Medical Group}

Background: We noted a low reported prevalence of delirium $(3 \%)$ in hospitalized older patients at a community teaching hospital in north central Wisconsin.

Purpose: This was a quality improvement project to report recognition of delirium by nurses before and after an educational intervention.

Methods: This project was performed on one medical unit in our hospital. Quality improvement data was collected at baseline and after the educational intervention. Data collected included observation by a geriatrician attending weekly interdisciplinary rounds to note any mention by nurses of delirium or confusion. The patient's electronic health record (EHR) was reviewed to note delirium assessment by "confusion assessment method for the intensive care unit (Vanderbilt)" (CAM-ICU) by the nurses for 2 days prior to the team meeting. The numbers of positive and total attempted CAMICU were recorded. Use of antipsychotics or benzodiazepines was reported as a "delirium marker." Diagnosis of delirium and dementia was obtained from the problem list in the EHR. The educational intervention included Just-in-Time Teaching during weekly Acute Care for Elders rounds during a 1-month period.

Results: In month 1, before intervention, CAM-ICU was performed 140 times in 2 days on 32 patients with an average CAM-ICU performed 2.2 times per patient/day. There were 3 concerning quotes for confusion during team rounds and 0 for delirium by nurses during team rounds. EHR review noted 7 patients had dementia, 2 had a positive CAM-ICU and 3 had a diagnosis of delirium. In month 2, after intervention, CAM-ICU was performed 163 times in 2 days on 35 patients with an average CAM-ICU performed 2.35 times per patient/ day. There were 6 concerning quotes regarding confusion and 1 regarding delirium by nurses during team rounds. EHR review noted 1 patient had dementia, 0 had a positive CAM-ICU and 0 patients with delirium diagnosis.

Conclusion: This quality improvement project using Justin-Time Teaching by a geriatrician during weekly rounds resulted in a modest increase in number of times CAMICU was performed, increased discussion of delirium during rounds, but no increase in delirium recognition using CAM-ICU. Areas for improvement include involving more physicians and nursing staff along with more structured delirium education.

\section{Stent Thrombosis: Regional Prevalence, Risk Factors, and Outcomes}

Andrew M. Ayers, Chi C. Cho, Robyn Shearer, M. Fuad Jan, Anjan Gupta

Aurora Cardiovascular Services, Aurora Health Care; Aurora Research Institute, Aurora Health Care

Background: Stent thrombosis is an infrequent but catastrophic complication of percutaneous coronary intervention (PCI). Many studies usually involve few stent thrombosis patients, generally less than 60 , given its prevalence. While dual antiplatelet therapy has decreased stent thrombosis significantly in the general population, there are still patients who present with occurrence and recurrence of stent thrombosis.

Purpose: We sought to define the prevalence of site-specific stent thrombosis in a larger cohort of patients by specific coronary territories and determine if this had an effect on cardiovascular outcomes. In addition we sought to elucidate the role of previous coronary artery bypass grafting (CABG) 
and subsequent PCI to determine if there is increased risk of stent thrombosis in specific post-CABG coronary artery territories and if these altered overall cardiovascular outcomes.

Methods: A retrospective review of our database on all patients presenting with stent thrombosis over the last 5 years was performed. Patients were included based on the accepted Academic Research Consortium definition of stent thrombosis.

Results: From January 2009 to February 2014, 220 patients were found to have had a stent thrombosis. Of these, 110 $(50.0 \%)$ had left anterior descending (LAD) artery lesions, $82(37.3 \%)$ had right coronary artery (RCA) lesions and $26(11.8 \%)$ had a stent thrombosis in the left circumflex artery (LCx). Prevalences of traditional risk factors were essentially equivalent regardless of which coronary artery developed stent thrombosis. All patients were on dual antiplatelet prior to developing stent thrombosis. Further analysis revealed $38(17.3 \%)$ had a prior history of CABG. A significant difference among the location of stent thrombosis and the history of CABG $(\mathrm{P}=0.043)$ was seen; $30.8 \%(\mathrm{n}=8)$ of patients with $\mathrm{LCx}$ stent thrombosis had prior $\mathrm{CABG}$ compared to $10.9 \%(n=12)$ and $22 \%(n=18)$ with LAD and RCA stent thrombosis, respectively.

Conclusion: In a large cohort of patients with stent thrombosis, LAD and RCA lesions were predominant, with LAD lesions representing half of all stent thromboses. PCI of these coronary territories thus infers a higher risk of stent thrombosis even in the presence of optimal medical therapy. Once stent thrombosis occurs, no significant difference in outcomes is seen based on location of the lesion alone. Additionally, patients who had prior CABG were significantly more likely to have stent thrombosis in the LCx and less likely in the LAD. This could be due to the fact that the left internal mammary artery graft is more often patent than vein grafts, which are more often anastomosed to the LCx and RCA and are at higher risk of needing stent placement after CABG.

\section{In Vitro Growth Suppression of Renal Carcinoma Cells by Curcumin}

Santhi D. Konduri, Madhavi Latha Yadav Bangaru, Phu Thanh Do, Shenglin Chen, Jeffrey Woodliff, Sanjay Kansra

\section{Aurora Research Institute, Aurora Health Care; Medical College of Wisconsin}

Background: Malignant clear cell renal carcinoma (ccRCC) is an aggressive tumor that is highly resistant to chemotherapy and radiation. Current therapeutic approaches to management of ccRCC have not significantly improved patient survival, therefore novel therapies are needed. The von Hippel-Lindau tumor suppressor gene is frequently mutated in ccRCC resulting in unregulated transcriptional activity of hypoxia-inducible factors (HIF) $1 \alpha$ and $2 \alpha$. HIFmediated transcription leads to increased growth factor expression and growth factor receptor (GFR)-mediated signaling. NFKB and STAT3 are phosphorylated in response to GFR activation and modulate gene expression, which promotes cell growth and invasion. Activated $\mathrm{NF \kappa B}$ and STAT3 expression is associated with ccRCC pathogenesis.

Purpose: The dietary polyphenol curcumin is a welldocumented antitumor agent and a known inhibitor of $\mathrm{NF} \kappa \mathrm{B}$ and STAT3 activation. Given the lack of effective therapies that block ccRCC progression, our objective was to examine whether curcumin could suppress the growth and migration of ccRCC cells, and whether this suppression was mediated via inhibition of NFKB and STAT3 activity.

Methods: Human ccRCC cell lines (769-p, 786-o, Caki-1, ACHN and A-498 cells) were exposed to curcumin to assess the impact of curcumin on ccRCC cell viability. To examine the mechanism by which curcumin induced cell death, we used 769-p cells, a highly aggressive human ccRCC cell line that does not express functional von Hippel-Lindau protein. The impact of curcumin on the phosphorylation status and transcriptional activity of NFKB and STAT3, in 769-p cells, was determined.

Results: Our results show that in ccRCC cells curcumin decreased cell proliferation and cell viability, abolished clonogenic property, induced apoptosis and blocked cellular migration. The growth suppressive and proapoptotic effects of curcumin were accompanied by decreased phosphorylation and transcriptional activity of NFאB and STAT3.

Conclusion: The ability of curcumin to induce apoptosis and inhibit migration of ccRCC cells justifies additional studies that explore the potential of developing curcumin or other $\mathrm{NF} \kappa \mathrm{B}$ and STAT3 inhibitors as novel therapeutic agents in the management of ccRCC.

\section{Triple Aim for Clinical Teachers (TACT): Faculty Physician Perceptions on Their Ability to Balance Clinical Quality, Trainee Learning, and Teaching Efficiency}

Minuja Muralidharan, Anne Getzin, Kjersti E. Knox, Bonnie L. Bobot, Marie M. Forgie, Nicole P. Salvo, Deborah Simpson

Departments of Internal Medicine, Family Medicine, Obstetrics and Gynecology, and Academic Affairs, Aurora UW Medical Group

Background: A common challenge facing teaching physicians is balancing high-quality student and resident teaching with efficient, high-quality care and patient service. Publicly accessible clinic performance reports increasingly affect where patients seek care and demand that teaching clinics rise to consumer expectations while training future physicians to function in the modern health care workplace. Limited information is available to guide physicians to achieve the triple aim for clinical teachers (TACT): clinical quality/patient experience, trainee learning, and teaching efficiency.

Purpose: To understand clinical teachers' TACT-related 